\title{
Micronutrientes na soja: produtividade e análise econômica
}

\author{
Micronutrients in soybean: productivity and economic analysis
}

\author{
Carlos Alberto Ceretta ${ }^{1}$ Aurélio Pavinato ${ }^{2}$ Paulo Sérgio Pavinato ${ }^{3}$ \\ Isabel Cristina Lopes Moreira ${ }^{4}$ Eduardo Girotto ${ }^{4}$ Éder Efraim Trentin ${ }^{4}$
}

\section{RESUMO}

A disponibilidade de produtos comerciais contendo micronutrientes tem aumentado nos últimos anos, embora existam resultados experimentais mostrando grande variabilidade, o que torna este tema contraditório. A diminuição do custo relativo no uso de micronutrientes e a expectativa de ganhos em escala, nos últimos anos, tem motivado produtores a utilizar micronutrientes como cobalto, boro e molibdênio, pela sua influência na fixação simbiótica de nitrogênio na soja. O objetivo do trabalho foi de determinar a viabilidade técnica $e$ econômica da aplicação de alguns micronutrientes, com destaque para Mo e Co, na produtividade de grãos da soja. O experimento foi conduzido nos anos agrícolas 2001/02 e 2002/ 03, na Fazenda Paineira, da SLC Agrícola Ltda, em Coronel Bicaco, RS, em Latossolo Vermelho distroférrico típico há oito anos sob plantio direto. O delineamento experimental foi de blocos ao acaso com três repetições, com parcelas de $10 \times 50 \mathrm{~m}$. A implantação da soja foi em 18/11 de 2001 e em 24/11 de 2002, com população de 225 e 190 mil plantas ha $a^{-1}$, para os dois anos agrícolas, respectivamente. $O$ espaçamento entre linhas foi de $0,40 \mathrm{~m}$ e a cultivar foi a RS-10 para os dois anos. Os tratamentos em 2001/02 foram: (1) CoMo; (2)CoMo+Mo; (3) $\mathrm{CoMo}+\mathrm{Mo}+\mathrm{Mo}$; (4) $\mathrm{CoMo}+\mathrm{Mo}+\mathrm{P30}$; (5) CoMo + B; (6) B; (7) Mo; (8) Mo+Mo; e (9) Testemunha. Em 2003/04, os tratamentos foram: (1) CoMo+2x Mo; (2)CoMo+2x Mo+B; (3) CoMo $+2 x$ $\mathrm{Mo}+$ Phitosol PK; (4) CoMo+2x Fortifol CaB; (5) CoMo $+2 x \mathrm{Mo}$ + LBE-PT1; (6) CoMo+2x Mo+P30; (7) CoMo+2x Mo+Stimulate; e (8) Testemunha. O uso de micronutrientes, especialmente Mo e Co, mostrou-se contraditório na avaliação da produtividade física de grãos através de comparação de médias. Entretanto, na maioria dos casos, o retorno econômico da aplicação dos micronutrientes foi positivo, mas evidenciou sua dependência de altas produtividades e preços favoráveis no momento da comercialização.

Palavras-chave: molibdênio, cobalto, boro, produtividade de soja.

\section{ABSTRACT}

The availability of commercial products containing micronutrients has increased in the latest years, but experimental results have shown great variability, turning this matter into something contradictory. The reduction in relative cost in the use o micronutrients and the expectancy of scale gain, in the latest years, have motivated producers to use micronutrients such as: cobalt, boron and molybdenum because of their influence in the symbiotic fixing of nitrogen in soybean. This work aimed at determining the technical and economic viability of the application of some micronutrients, emphasizing Mo and Co, in soybean productiveness. The experiment was carried out in the agricultural years of 2001/ 02 and 2002/03, at Paineira Farm, from SLC Agrícola Ltda, in Coronel Bicaco, RS, in a Hapludox and it had been under no tillage for eight years. The experimental outlining was of random blacks with three repetitions, with portions of $10 \mathrm{x}$ $15 \mathrm{~m}$. Soybean implantation was on $11 / 18 / 2001$ and on 11/ 24/2002, with 225 and 190 thousand plants ha $^{-1}$, for the two agricultural years respectively. The spacing between lines was of $0,40 \mathrm{~m}$ and the variety was RS-10 for the two years. Treatments in 2001/02 were: (1) CoMo; (2) CoMo + Mo; (3) $\mathrm{CoMo}+\mathrm{Mo}+\mathrm{Mo}$; (4) CoMo + Mo + P30; (5) CoMo + B; (6) B; (7) Mo; (8) Mo + Mo; and (9) Testemunha. In 2002/03 treatments were: (1) CoMo + 2x Mo; (2) CoMo $+2 x \mathrm{Mo}+\mathrm{B}$; (3) CoMo $+2 x$ Mo + Phitosol PK; (4) CoMo $+2 x$ Fortifol CaB; (5) CoMo + 2xMo + LBE-PT1; (6) CoMo + 2x Mo +

${ }^{1}$ Engenheiro Agrônomo, Doutor, Professor Titular, Universidade Federal de Santa Maria (UFSM), Departamento de Solos, 97105900, Santa Maria, RS, Brasil. E-mail: ceretta@ccr.ufsm.br Bolsista CNPq. Autor para correspondência.

${ }^{2}$ Engenheiro Agrônomo, Mestre em Agronomia, SLC Agrícola Ltda, Brasil. E-mail: pavinato@terra.com.br

${ }^{3}$ Engenheiro Agrônomo, Doutorando do Programa de Pós-graduação em Ciência do Solo, Faculdade de Ciências Agronômicas, Universidade Estadual de São Paulo, Brasil. E-mail: pspavinato@fca.unesp.br

${ }^{4}$ Acadêmicos do Curso de Agronomia da UFSM e bolsistas de iniciação científica, Brasil. 
P30; (7) CoMo + 2x Mo + Stimulate; and (8) Testemunha. The use of micronutrients, especially Mo and Co, has shown to be a contradictory one in the physical productiveness evaluation of the grains through the comparison of averages. However, in most cases, the economic return of the micronutrient application was positive, but it showed its dependence on high productiveness and favorable prices at the moment of commercialization.

Key words: molybdenum, cobalt, boron, soybean produtivity.

\section{INTRODUÇÃO}

A disponibilidade de produtos comerciais contendo micronutrientes tem aumentado nos últimos anos, e existem resultados experimentais mostrando grande variabilidade de resposta à sua aplicação. Por outro lado, o aumento na produtividade da soja e, por conseqüência, a diminuição do custo relativo no uso de micronutrientes e a expectativa de ganhos em escala tem motivado produtores a utilizar micronutrientes como cobalto, boro e, principalmente molibdênio, pela sua influência na fixação simbiótica de nitrogênio na soja.

As respostas a micronutrientes têm sido mais freqüentes nas condições do Cerrado, onde BROCH \& FERNANDES (1999) mostraram que na média de 12 estudos com micronutrientes aplicados via sementes, houve aumento de até 6,5 sacas ha-1 na $^{-1}$ produtividade da soja. No entanto, no sul do Brasil a resposta positiva a micronutrientes na produtividade depende muito da combinação de uma série de fatores, não bem compreendidos por insuficiente número de experimentos realizados. Por isso, há a necessidade de mais estudos que auxiliem técnicos e produtores na sua tomada de decisão sobre o uso de micronutrientes, embora se deva considerar que este tema sempre será objeto de discussão para cada situação, evitando-se generalizações.

O molibdênio, cobalto e boro são nutrientes importantes à fixação biológica do nitrogênio na soja, cultura esta que representa a principal fonte de receita de grande parte dos agricultores e é responsável pelo ingresso de divisas no Brasil pelas exportações. Na planta, o molibdênio participa como cofator integrante nas enzimas nitrogenase, redutase do nitrato e oxidase do sulfato, e está intimamente relacionado com o transporte de elétrons durante as reações bioquímicas das plantas (PRICE et al., 1972; LANTMANN, 2002). O cobalto é um nutriente necessário para a síntese da cobalamina (Vitamina B12), que participa dos passos metabólicos para a formação da leghemoglobina, cuja afinidade com o oxigênio é elevada, e regula sua concentração nos nódulos impedindo a inativação da enzima nitrogenase (FAVARIN \& MARINI, 2000). O boro, embora não tenha ação direta sobre a fixação biológica, é um elemento que ativa a enzima fosforilase do amido, responsável pela síntese de amido, substância de reserva das sementes, raízes, tubérculos e colmos (FAVARIN \& MARINI, 2000).

O objetivo do trabalho foi determinar a viabilidade técnica e econômica da aplicação de alguns micronutrientes, com destaque para Mo e Co, na produtividade de grãos da soja.

\section{MATERIALEMÉTODOS}

O experimento foi conduzido nos anos agrícolas 2001/02 e 2002/03, na Fazenda Paineira, da SLC Agrícola Ltda, em Coronel Bicaco, RS, em um Latossolo Vermelho distroférrico típico, que se caracteriza como solo profundo, avermelhado, textura argilosa e com presença de óxidos. A área onde o experimento foi instalado estava há oito anos sob plantio direto.

Os teores de fósforo e potássio no solo enquadravam-se na classe muito alto e alto, respectivamente, antes da instalação do experimento nos dois anos agrícolas (Tabela 1), enquanto que cálcio e o magnésio estavam altos, com saturação com bases alta e com alumínio mínima (CFS RS/SC, 1994).

O delineamento experimental foi de blocos ao acaso com três repetições, com parcelas de 10 x 50m. A

Tabela 1 - Caracterização do solo no momento da implantação do experimento nos anos agrícolas 2001/02 e 2002/03 na profundidade de 0 a $10 \mathrm{~cm}$.

\begin{tabular}{|c|c|c|c|c|c|c|c|c|c|}
\hline \multirow{2}{*}{ Ano } & Argila & $\mathrm{pH}-\mathrm{H}_{2} \mathrm{O}$ & Índice & $\mathrm{P}$ & $\mathrm{K}$ & M.O. & $\mathrm{Al}$ & $\mathrm{Ca}$ & $\mathrm{Mg}$ \\
\hline & $\mathrm{g} \mathrm{kg}^{-1}$ & $1: 1$ & SMP & \multicolumn{2}{|c|}{$\mathrm{mg} \mathrm{dm}{ }^{-3}$} & $\mathrm{~g} \mathrm{~kg}^{-1}$ & \multicolumn{3}{|c|}{$\mathrm{cmol}_{\mathrm{c}} \mathrm{dm}^{-3}$} \\
\hline $2001 / 02$ & 700 & 5,3 & 6,1 & 10,0 & 98 & 36,0 & 0,1 & 6,5 & 3,0 \\
\hline $2002 / 03$ & 700 & 5,9 & 6,1 & 15,0 & +200 & 43,0 & 0,0 & 6,7 & 2,8 \\
\hline \multirow{2}{*}{ Ano } & \multicolumn{2}{|c|}{$\mathrm{H}+\mathrm{Al}$} & \multicolumn{3}{|c|}{ CTC $\left(\mathrm{cmol}_{\mathrm{c}} \mathrm{dm}^{-3}\right)$} & \multicolumn{4}{|c|}{ Saturação (\%) } \\
\hline & \multicolumn{2}{|c|}{$\mathrm{cmol}_{\mathrm{C}} \mathrm{dm}^{-3}$} & Efetiva & \multicolumn{2}{|c|}{$\mathrm{pH} \mathrm{7,0}$} & \multicolumn{2}{|c|}{ Alumínio } & \multicolumn{2}{|c|}{ Bases } \\
\hline $2001 / 02$ & \multicolumn{2}{|c|}{3,3} & 13,1 & \multicolumn{2}{|c|}{16,4} & \multicolumn{2}{|c|}{1} & \multicolumn{2}{|c|}{75} \\
\hline $2002 / 03$ & \multicolumn{2}{|c|}{3,3} & 10,1 & \multicolumn{2}{|c|}{13,3} & \multicolumn{2}{|c|}{0} & \multicolumn{2}{|c|}{75} \\
\hline
\end{tabular}


implantação da soja no primeiro ano foi em 18/11/2001 com população de aproximadamente 225 mil plantas ha ${ }^{-1}$ e espaçamento entre linhas de 0,40m. A implantação da soja no segundo ano foi em 24/11/2002 com população de 190 mil plantas ha ${ }^{-1}$ com igual espaçamento. A cultivar utilizada nos dois anos agrícolas foi a RS-10.

Os tratamentos à soja cultivada em 2001/ 02 foram as seguintes combinações: (1) CoMo; (2) CoMo + Mo; (3) CoMo + Mo + Mo; (4) CoMo + Mo + P30; (5) CoMo + B; (6) B; (7) Mo; (8) Mo + Mo; e (9) Testemunha. Os produtos utilizados nestas combinações foram: para os tratamentos com CoMo, foi usado o produto comercial CoMo Plus 250 (1,7\% de Co e 17\% de Mo) na dose de 0,091 ha $^{-1}$ aplicado via semente; nos tratamentos onde Mo foi usado separadamente, a fonte utilizada foi o Molibdato de Sódio (39,5\% de Mo) na dose de 0,12kg ha $^{-1}$, aplicado via foliar juntamente com herbicidas aos 30 dias após emergência (DAE), e quando em duas vezes, também aos 60 DAE; o P30 (30\% de P, 5\% de N e $1,2 \%$ de $\mathrm{Mg}$ ) foi usado na dose de 2,0 $1 \mathrm{ha}^{-1}$ aplicado aos $60 \mathrm{DAE}$; o B foi aplicado com o produto comercial Solubor (20,5\% de B) na dose de 1,0 $\mathrm{kg} \mathrm{ha}^{-1}$, aplicado via foliar, aos 60 DAE da soja.

No cultivo da soja, em 2002/03, houve algumas mudanças nos tratamentos, mas manteve-se a prioridade na avaliação do uso de Mo, Co e B. Os tratamentos foram constituídos de: (1) CoMo + Mo + $\mathrm{Mo}$; (2) $\mathrm{CoMo}+\mathrm{Mo}+\mathrm{Mo}+\mathrm{B}$; (3) $\mathrm{CoMo}+\mathrm{Mo}+\mathrm{Mo}+$ Phitosol PK; (4) CoMo + 2x Fortifol CaB; (5) CoMo + $\mathrm{Mo}+\mathrm{Mo}+\mathrm{LBE}-\mathrm{PT} 1 ;$ (6) $\mathrm{CoMo}+\mathrm{Mo}+\mathrm{Mo}+\mathrm{P} 30$; (7) $\mathrm{CoMo}+\mathrm{Mo}+\mathrm{Mo}+$ Stimulate; e (8) Testemunha. Nos tratamentos com CoMo, foi utilizado o produto comercial CoMo Plus 250 (1,7\% de Co e $17 \%$ de Mo) na dose de 0,09 $\mathrm{l} \mathrm{ha}^{-1}$ aplicado via semente. Quando o Mo foi aplicado separadamente, a fonte utilizada foi o Molibdato de Sódio (39,5\% de Mo), na dose foi de $0,12 \mathrm{~kg} \mathrm{ha}^{-1}$, aplicado via foliar aos 30 e 60 DAE. A fonte de B foi o produto comercial Solubor (20,5\% de B) na dose de 1,0kg ha ${ }^{-1}$, aplicado via foliar aos 60 DAE. No tratamento com Phitosol PK, usou-se o produto comercial de mesmo nome ( $30 \%$ de $\mathrm{P}_{2} \mathrm{O}_{5}$ e $20 \%$ de $\mathrm{K}_{2} \mathrm{O}$ ), na dose de 2,0 $1 \mathrm{ha}^{-1}$, aplicado via foliar aos $30 \mathrm{DAE}$. O Fortifol CaB (10\% de Ca e 1\% de B), foi usado na dose de 0,2 1 ha $^{-1}$ e aplicado via foliar aos 60 DAE e no estádio R1. O LBE-PT1 (4\% de N, 16\% de $\mathrm{P}_{2} \mathrm{O}_{5}$ e 1\% de $\mathrm{K}_{2} \mathrm{O}$ ) foi aplicado na dose de $0,1 \mathrm{l} \mathrm{ha}{ }^{-1}$, aos $30 \mathrm{DAE}$. O P30 (30\% de P, $5 \%$ de $\mathrm{N}$ e 1,2\% de $\mathrm{Mg}$ ), foi usado na dose de 2,0 $1 \mathrm{ha}^{-1}$ e aplicado 60 DAE. O Stimulate (4\% de Mo) foi aplicado na dose de $0,251 \mathrm{ha}^{-1}$, aos $90 \mathrm{DAE}$.

A produção de matéria seca foi obtida através da coleta da parte aérea das plantas em pleno florescimento e as amostras constituídas de três sub amostras de um metro linear, coletadas aleatoriamente na área útil das parcelas. Depois de secas em estufa a $65^{\circ} \mathrm{C}$ até peso constante, as amostras foram moídas em triturador de forrageiras e após, em moinho Willey ( $<40$ mesh).

As quantidades acumuladas de nitrogênio, potássio e fósforo no tecido vegetal foram determinadas segundo a metodologia descrita em TEDESCO et al. (1995). A produtividade de grãos da soja foi avaliada em uma área de $244 \mathrm{~m}^{2}$ por parcela, que correspondeu a uma passada de colheitadeira com plataforma de 4,88m de largura ao longo dos 50m de comprimento da parcela, colhendo uma faixa central. A produtividade de grãos foi corrigida para zero de impurezas e $13 \%$ de umidade.

$\mathrm{Na}$ análise econômica, o cálculo dos custos com a aplicação dos micronutrientes foi efetuado levando-se em consideração os valores de compra dos produtos contendo micronutrientes, no mês de novembro dos anos de 2001 e 2002. Os valores foram convertidos para dólar (US\$), considerando a conversão média do dólar nos respectivos meses. A variação na receita obtida com a aplicação dos micronutrientes foi realizada levando em conta o valor de venda da soja no mês de junho em 2002 e 2003, mês no qual, normalmente, o produtor realiza a comercialização da sua produção na região Sul. Os valores também foram expressos em dólar e a conversão realizada pela média nos respectivos meses.

Os resultados de produção de matéria seca, nutrientes acumulados no tecido e produtividade de grãos da soja foram submetidos à análise da variância e teste de Duncan a 5\% de probabilidade de erro.

\section{RESULTADOS E DISCUSSÃO}

A produção de matéria seca e as quantidades de $\mathrm{N}, \mathrm{P}$ e $\mathrm{K}$ acumuladas até o estádio de pleno florescimento da soja não foram alteradas com a aplicação dos produtos nos dois anos agrícolas (Tabela 2). A maior população de plantas é uma das justificativas para a produção média de matéria seca superior em 17,3\% no ano agrícola 2001/02. Contudo, a menor produção de matéria seca em 2002/03 não se refletiu na produtividade média de grãos, que foram muito semelhantes nos dois anos agrícolas (Tabela 2). Isso pode ser um indicativo de que a utilização de menores populações de plantas, que está sendo uma tendência nos últimos anos, pode ser considerada para gerar plantas mais robustas e resistentes a doenças, aproveitando assim o potencial produtivo de cada cultivar.

O acúmulo médio de nitrogênio no tecido das plantas de soja foi de 167 e $149 \mathrm{~kg} \mathrm{ha}^{-1}$ em 2001/02 e 2002/03, respectivamente e isso pode ser justificado pela maior produção de matéria seca neste ano, pois a 
Tabela 2 - Matéria seca da parte aérea no pleno florescimento, nutrientes acumulados no tecido e produtividade de grãos da soja, sob a aplicação de micronutrientes nos anos agrícolas 2001/02 e 2002/03.

\begin{tabular}{|c|c|c|c|c|c|}
\hline \multirow{2}{*}{ Tratamento } & \multirow{2}{*}{ Matéria seca } & \multicolumn{3}{|c|}{ Nutrientes no tecido } & \multirow{2}{*}{ Produtividade de grãos } \\
\hline & & $\mathrm{N}$ & $\mathrm{P}$ & $\mathrm{K}$ & \\
\hline \multicolumn{6}{|c|}{ Ano agrícola 2001/02 } \\
\hline \multicolumn{6}{|c|}{$\mathrm{kg} \mathrm{ha}^{-1}$} \\
\hline CoMo & $9.278^{\mathrm{ns}}$ & $193^{\mathrm{ns}}$ & $27,3^{\mathrm{ns}}$ & $215^{\mathrm{ns}}$ & $3.227 b^{*}$ \\
\hline CoMo+Mo & 8.969 & 175 & 26,9 & 225 & $3.359 \mathrm{ab}$ \\
\hline $\mathrm{CoMo}+\mathrm{Mo}+\mathrm{Mo}$ & 8.589 & 173 & 26,1 & 203 & $3.596 \mathrm{a}$ \\
\hline $\mathrm{CoMo}+\mathrm{Mo}+\mathrm{P} 30$ & 7.969 & 154 & 26,2 & 205 & $3.363 \mathrm{ab}$ \\
\hline $\mathrm{CoMo}+\mathrm{B}$ & 8.719 & 170 & 29,5 & 227 & $3.273 \mathrm{~b}$ \\
\hline B & 8.900 & 179 & 29,3 & 223 & $3.453 \mathrm{ab}$ \\
\hline Mo & 7.542 & 144 & 25,7 & 194 & $3.260 \mathrm{~b}$ \\
\hline $\mathrm{Mo}+\mathrm{Mo}$ & 7.946 & 151 & 23,9 & 193 & $3.206 \mathrm{~b}$ \\
\hline Testemunha & 8.113 & 166 & 25,9 & 197 & $3.273 \mathrm{~b}$ \\
\hline Média & 8.447 & 167 & 26,8 & 209 & 3.334 \\
\hline CV (\%) & 11,41 & 15,78 & 16,88 & 12,44 & 3,97 \\
\hline \multicolumn{6}{|c|}{ Ano agrícola 2002/03 } \\
\hline $\mathrm{CoMo}+2 \mathrm{x} \mathrm{Mo}$ & $7.567^{\mathrm{ns}}$ & $156^{\mathrm{ns}}$ & $29,0^{\mathrm{ns}}$ & $246^{\mathrm{ns}}$ & $3.510 \mathrm{ab}^{*}$ \\
\hline $\mathrm{CoMo}+2 \times \mathrm{Mo}+\mathrm{B}$ & 8.250 & 155 & 30,9 & 266 & 3.433 bc \\
\hline CoMo+2x Mo+Phit. PK & 6.967 & 141 & 28,3 & 233 & $3.696 \mathrm{a}$ \\
\hline $\mathrm{CoMo}+2 \times \mathrm{Mo}$ Fortifol $\mathrm{CaB}$ & 6.850 & 149 & 27,5 & 232 & 3.344 bc \\
\hline CoMo+2x Mo+LBE-PT1 & 7.367 & 154 & 29,3 & 238 & $3.473 \mathrm{ab}$ \\
\hline $\mathrm{CoMo}+2 \mathrm{x} \mathrm{Mo}+\mathrm{P} 30$ & 6.800 & 152 & 29,3 & 221 & $3.470 \mathrm{ab}$ \\
\hline $\mathrm{CoMo}+2 \mathrm{x} \mathrm{Mo+Stimulate}$ & 6.600 & 135 & 29,4 & 225 & $3.340 \mathrm{bc}$ \\
\hline Testemunha & 7.208 & 153 & 29,4 & 241 & 3.184 c \\
\hline Média & 7.201 & 149 & 29,1 & 238 & 3.431 \\
\hline CV (\%) & 12,64 & 18,62 & 11,64 & 10,82 & 3,97 \\
\hline
\end{tabular}

${ }^{\text {ns }}$ Não significativo na mesma coluna para cada ano agrícola.

* Médias seguidas pela mesma letra na coluna e para cada ano agrícola não diferem pelo teste Duncan a 5\% de probabilidade de erro.

concentração média de $\mathrm{N}$ no tecido foi semelhante nos dois anos, sendo de 2,00 e 2,08\% em 2001/02 e 2002/03, respectivamente. A maior acumulação de P no segundo ano foi conseqüência da maior concentração média de $\mathrm{P}$ no tecido, que foi de $0,41 \%$ contra os $0,34 \%$ no primeiro ano. Além disso, os acúmulos de $\mathrm{P}$ e K podem ter sido favorecidos, no segundo ano, pelos maiores teores destes nutrientes no solo, conforme a análise do solo das duas áreas experimentais (Tabela 1).

A produtividade de grãos foi alta nos dois anos agrícolas, pois variou de 3.206 a $3.596 \mathrm{~kg} \mathrm{ha}^{-1} \mathrm{em}$ 2001/02 e de 3.184 a 3.696 $\mathrm{kg} \mathrm{ha}^{-1}$ em 2002/03. Em 2001/02 a produtividade foi incrementada somente quando foram aplicados CoMo via semente e também mais duas aplicações de Mo via foliar aos 30 e 60 dias após a emergência das plantas. Entretanto, deve-se observar que a produtividade foi a mesma quando foi aplicado CoMo na semente e apenas uma aplicação de Mo em cobertura aos 30 dias após emergência. Na interpretação dos dados, deve-se considerar as comparações como resultantes dos efeitos dos tratamentos, mas também do teste de comparação de médias utilizado e o nível de significância, pois se pode constatar que existem tratamentos com CoMo cuja produtividade de grãos não difere de quando foi aplicado apenas boro ou mesmo não foi aplicado CoMo. Tanto que houve quatro tratamentos com CoMo e mais dois apenas com Mo que proporcionaram produtividade de grãos semelhante à testemunha. Em 2002/03 a interpretação da resposta ao uso de Mo também deve ser criteriosa porque em três tratamentos onde foi utilizado CoMo via semente e mais duas aplicações de Mo em cobertura a produtividade de grãos de soja não foi maior do que a testemunha. Não é possível destacar algum produto associado ao CoMo + 2x Mo, porque se por um lado o acréscimo obtido com a adição de Phitosol PK fez com que a produtividade se destacasse em relação a adição de Fortifol CaB e Stimulate, o mesmo não proporcionou acréscimo na produtividade de grãos quando comparado ao uso exclusivo de CoMo $+2 \mathrm{x}$ Mo. 
Os resultados dos dois anos agrícolas podem ser agregados à observação de BORKERT (2002), o qual cita que, em geral, vários trabalhos de pesquisa com micronutrientes feitos no RS e em outros estados do Brasil mostram ausência de resposta à aplicação de micronutrientes na grande maioria das situações. As justificativas para estes resultados são a capacidade adequada de suprimento pelos solos, em função de sua origem, e a presença de contaminantes contendo micronutrientes em corretivos e fertilizantes (BISSANI \& GIANELLO, 2003). Entretanto, aumento de produtividade de grãos de até $15 \%$ foi atribuído à aplicação de Mo via semente através do uso de várias fórmulas de produtos comerciais (SFREDO et al., 1997). Os mesmos autores atribuíram também importância ao Co, pois estes dois nutrientes fazem parte do ciclo do nitrogênio na cultura e influenciam no aumento observado do teor de proteína no grão. Outros dados mostram que o Mo promoveu acréscimo na produtividade de grãos de soja em solos com $\mathrm{pH}$ inferior a 5,5, tendo havido deficiência de nitrogênio no início do ciclo de desenvolvimento da cultura (BISSANI \& GIANELLO, 2003). Contudo, o pH do solo não deve ser um indicador utilizado isoladamente quando sob plantio direto, porque as condições do solo modificam-se em relação aos sistemas com revolvimento de solo, além do que o pH 5,5 é a meta a ser atingida no plantio direto, ou seja, em muitos casos o pH do solo estará inferior a 5,5 não significando necessariamente com isso probabilidade de resposta ao Mo. O pH (água 1:1) dos solos utilizados era 5,3 e 5,9 nos dois solos e isso pode justificar a menor probabilidade de resposta ao Mo, pois QUAGGIO et al. (1998) observou resposta positiva da soja à aplicação de Mo na semente até $\mathrm{pH}$ $5,2\left(\mathrm{CaCl}_{2}\right)$, enquanto que LANTMANN et al. (1989) observaram que o Mo ficava mais disponível em $\mathrm{pH}$ 4,8 $\left(\mathrm{CaCl}_{2}\right)$ e que acima disso não era necessário aplicar Mo nas sementes de soja, desde que houvesse disponibilidade de Mo no solo.

É comum na literatura observar-se resultados diversos sobre o uso de micronutrientes. Um levantamento realizado em vários estados brasileiros com 35 culturas, mostrou deficiência de boro em 24 culturas, de zinco em 23, de molibdênio e cobre em 7 e manganês em 6 culturas (FAVARIN \& MARINI, 2000). Os mesmos autores ressaltam que houve uma freqüência de resposta de $36 \%$ para o zinco, $24 \%$ para o boro, $14 \%$ para o molibdênio, $12 \%$ para o cobre e $8 \%$ para o manganês nas culturas anuais como soja, milho, arroz, não diferindo substancialmente nas culturas perenes, exceto o molibdênio cuja freqüência de resposta foi de $21 \%$. Contudo, a discussão sobre o uso de micronutrientes deve ser avaliada regionalmente, considerando-se o tipo e manejo do solo, clima e culturas implantadas, tendo-se especial cuidado com o excesso ou má distribuição do calcário em muitas situações de lavouras (PAULETTI, 1998).

Na discussão sobre o uso de micronutrientes na soja, não basta apenas a avaliação da produtividade física, devendo ser agregada a análise econômica, porque estas variáveis são fundamentais à tomada de decisão de produtores e técnicos.

A análise econômica do cultivo da soja no ano agrícola 2001/02 evidenciou que a produtividade de grãos de $3.596 \mathrm{kgha}^{-1}$, obtida com a aplicação de CoMo via semente mais Mo aos 30 e 60 dias após a emergência, representou um acréscimo em torno de $10 \%$ na produtividade de grãos, aumentando $323 \mathrm{~kg}$ $\mathrm{ha}^{-1}$ em relação à testemunha, o que significou um retorno líquido de US\$ 49,19 por hectare (Tabela 3). Contudo, a interpretação deve ser cuidadosa porque o retorno líquido foi negativo em dois tratamentos onde foi utilizado CoMo via semente e também onde foi somente Mo em cobertura em uma ou duas aplicações.

Na safra de 2002/03, a aplicação de todos os produtos resultou em retorno líquido positivo, especialmente porque o preço da saca de soja atingiu US\$ 11,55 em junho, mês tomado como referência para a venda do produto. Isso representou um aumento de cerca de $18 \%$ em relação à safra 2002, quando a saca era cotada a US\$9,82 no mesmo mês. Além disso, a produtividade média na safra 2002/03 foi 3\% maior, equivalendo a um acréscimo de $97 \mathrm{~kg} \mathrm{ha}^{-1}$, e um aumento no retorno médio de US\$18,67 por hectare em relação a safra de 2002. Isso mostra que as vantagens econômicas no uso dos micronutrientes utilizados neste trabalho, dependem muito da obtenção de altas produtividades, bem como preços compensatórios à venda dos grãos. Destaque neste ano foi obtido com a aplicação de CoMo via semente mais duas aplicações foliares de Mo e ainda a aplicação do produto Phitosol PK, o que representou um acréscimo no retorno líquido de US\$ 87,22 ha-1, em relação à testemunha, o que representa incremento no lucro de $47 \%$, tomando-se por base o custo de produção médio de 35 sacas ha-1.

\section{CONCLUSÕES}

O uso de micronutrientes, especialmente Mo e Co, mostrou-se contraditório na avaliação da produtividade física de grãos através do uso de teste de comparação de médias. Entretanto, na maioria dos casos, o retorno econômico da aplicação dos micronutrientes utilizados foi positivo, mas evidenciou sua dependência de altas produtividades e preços favoráveis no momento da comercialização. 
Tabela 3 - Viabilidade econômica, em US\$, da aplicação de micronutrientes e outros produtos comerciais na produtividade de grãos da soja, quando comparada com a testemunha, nos anos agrícolas 2001/02 e 2002/03*.

\begin{tabular}{|c|c|c|c|}
\hline \multirow[t]{2}{*}{ Tratamento } & $\begin{array}{l}\text { Custo aplicação dos } \\
\text { micronutrientes** }\end{array}$ & $\begin{array}{c}\text { Acréscimo no retorno } \\
\text { econômico }\end{array}$ & Retorno líquido \\
\hline & \multicolumn{3}{|c|}{ Ano agrícola 2001/02 (U\$ ha $\left.{ }^{-1}\right)$} \\
\hline CoMo & 0,90 & $-7,53$ & $-8,43$ \\
\hline $\mathrm{CoMo}+\mathrm{Mo}$ & 1,53 & 14,08 & 12,55 \\
\hline $\mathrm{CoMo}+\mathrm{Mo}+\mathrm{Mo}$ & 3,67 & 52,86 & 49,19 \\
\hline $\mathrm{CoMo}+\mathrm{Mo}+\mathrm{P} 30$ & 4,91 & 14,73 & 9,82 \\
\hline $\mathrm{CoMo}+\mathrm{B}$ & 4,20 & 0,00 & $-4,20$ \\
\hline B & 3,30 & 29,46 & 26,16 \\
\hline Mo & 0,63 & $-2,13$ & $-2,76$ \\
\hline \multirow[t]{2}{*}{$\mathrm{Mo}+\mathrm{Mo}$} & 2,77 & $-10,97$ & $-13,74$ \\
\hline & \multicolumn{3}{|c|}{ Ano agrícola 2002/03 (U\$ ha ${ }^{-1}$ ) } \\
\hline $\mathrm{CoMo}+2 \mathrm{x} \mathrm{Mo}$ & 3,80 & 62,76 & 58,96 \\
\hline $\mathrm{CoMo}+2 \mathrm{x} \mathrm{Mo+B}$ & 5,20 & 48,13 & 42,93 \\
\hline CoMo+2x Mo+Phit.PK & 11,34 & 98,56 & 87,22 \\
\hline $\mathrm{CoMo}+2 \mathrm{x} \mathrm{Mo+Fortifol} \mathrm{CaB}$ & 3,30 & 30,99 & 27,69 \\
\hline CoMo+2x Mo+LBE-PT1 & 7,99 & 55,83 & 47,84 \\
\hline $\mathrm{CoMo}+2 \mathrm{x} \mathrm{Mo}+\mathrm{P} 30$ & 4,81 & 55,25 & 50,44 \\
\hline $\mathrm{CoMo}+2 \mathrm{x} \mathrm{Mo+Stimulate}$ & 10,31 & 30,22 & 19,91 \\
\hline
\end{tabular}

* Foi considerado o preço médio da saca de soja de US\$ 9,82 em junho de 2002 e US\$ 11,55 em junho de 2003.

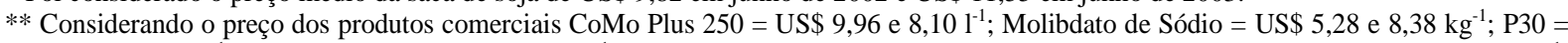
US\$ 0,94 e 0,50 $\mathrm{l}^{-1}$; Solubor = US\$ 1,80 e 1,40 kg-1, para os anos de 2001/02 e 2002/03, respectivamente; Phitosol PK = US\$ 3,77 $\mathrm{l}^{-1}$; Fortifol CaB = US\$ 1,12 l $\mathrm{l}^{-1}$; LBE-PT1 = US\$ 41,90 l $\mathrm{l}^{-1}$; e Stimulate = US\$ 21,79 $\mathrm{l}^{-1}$. Incluído o custo de aplicação de US\$ 1,50 e 1,06 ha ${ }^{-1}$ para cada aplicação aos 60 ou 90 DAE em 2001/02 e 2002/03, respectivamente. Valores obtidos considerando a conversão do dólar sendo equivalente a R\$ 2,54 e 3,58 para o mês de novembro dos anos de 2001 e 2002, respectivamente.

\section{REFERÊNCIAS BIBLIOGRÁFICAS}

BISSANI, C.A.; GIANELLO, C. Utilização de micronutrientes. In: CURSO DE FERTILIDADE DO SOLO EM PLANTIO DIRETO, 6., 2003, Passo Fundo. Palestras... Passo Fundo : Aldeia Norte, 2003. p.52-63.

BORKERT, C.M. Ganhos em produtividade de culturas anuais com micronutrientes na Região Sul. In: CURSO DE FERTILIDADE DO SOLO EM PLANTIO DIRETO, 5., 2002, Guarapava. Resumos de palestras... Passo Fundo : Aldeia Norte, 2002. p.81-96.

BROCH, D.L.; FERNANDES, C.H. Resposta da soja à aplicação de micronutrientes. Maracaju, MS : Fundação MS, 1999. 56p. (Informativo Técnico 02/99).

COMISSÃO DE FERTILIDADE DO SOLO RS/SC (CFS RS/ $\mathrm{SC}$ ). Recomendações de adubação e calagem para os estados do Rio Grande do Sul e Santa Catarina. 3.ed. Passo Fundo : SBCS/NRS, 1994. 223p.

FAVARIN, J.L.; MARINI, J.P. Importância dos micronutrientes para a produção de grãos. In: SOCIEDADE NACIONAL DA AGRICULTURA, 2000. Acesso em: 04/2003. On line. Disponível na Internet: www.sna.com.br.

LANTMANN, A.F. et al. Resposta da soja a molibdênio em diferentes níveis de $\mathrm{pH}$ do solo. Revista Brasileira de Ciência do Solo, Campinas, v.13, n.1, p.45-49, 1989.
LANTMANN, A.F. Nutrição e produtividade da soja com molibdênio e cobalto. Artigos EMBRAPA, Coletânea rumos \& debates, 2002. Acesso em: 04/2003. On line. Disponível em <www.embrapa.org.br >

PAULETTI, V. Disponibilidade e resposta de culturas a micronutrientes em solos arenosos. In: FRIES, M.R. (Coord). Plantio direto em solos arenosos: alternativas de manejo para a sustentabilidade agropecuária. UFSM/Departamento de Solos. Santa Maria : Palotti, 1998. p.82-102.

PRICE, C.A. et al. Functions of micronutrients in plants. In: MONTVEDT, J.J. et al. (eds). Micronutrients in agriculture; Zn, Fe, Mo, Cu, B, Mn. Madison : Soil Science Society of America, 1972. p.231-242.

QUAGGIO, J.A. et al. Isoquantas de produtividade de soja e sorgo para níveis de calagem e molibdênio. Revista Brasileira de Ciência do Solo, Campinas, v.22, p.337344, 1998.

SFREDO, G.J. et al. Eficácia de produtos contendo micronutrientes, aplicados via semente, sobre produtividade e teores de proteína na soja. Revista Brasileira de Ciência do Solo, Campinas. v.21, p.41-45, 1997.

TEDESCO, M.J. et al. Análises de solo, plantas e outros materiais. 2.ed. Porto Alegre : UFRGS, Faculdade de Agronomia, 1995. 174p. 